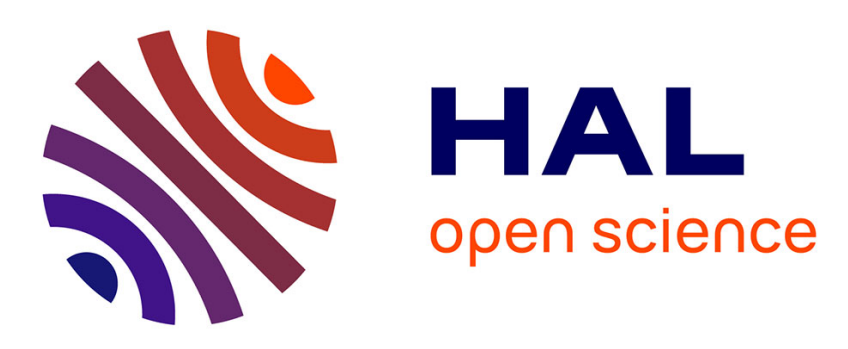

\title{
On the Existence of the Normal Form for Nonlinear Delay Systems
}

Claudia Califano, Claude H. Moog

\section{To cite this version:}

Claudia Califano, Claude H. Moog. On the Existence of the Normal Form for Nonlinear Delay Systems. Malisoff, M.; Pepe, P.; Mazenc, F.; Karafyllis, I. Recent Results on Nonlinear Delay Control Systems, 4, Springer, pp.113-142, 2016, Advances in Delays and Dynamics, 9783319180717. 10.1007/978-3319-18072-4_6. hal-01179602

\section{HAL Id: hal-01179602 \\ https://hal.science/hal-01179602}

Submitted on 27 Apr 2021

HAL is a multi-disciplinary open access archive for the deposit and dissemination of scientific research documents, whether they are published or not. The documents may come from teaching and research institutions in France or abroad, or from public or private research centers.
L'archive ouverte pluridisciplinaire HAL, est destinée au dépôt et à la diffusion de documents scientifiques de niveau recherche, publiés ou non, émanant des établissements d'enseignement et de recherche français ou étrangers, des laboratoires publics ou privés. 


\title{
On the Existence of the Normal Form for Nonlinear Delay Systems
}

\author{
Claudia Califano and Claude H. Moog
}

\begin{abstract}
The normal form is discussed for nonlinear systems affected by constant commensurate delays. Two different forms are argued. In particular, necessary and sufficient conditions are given under which a nonlinear time-delay system can be decomposed into a (weakly) observable subsystem and a non observable subsystem. Whenever such a decomposition exists, additional conditions are required to ensure the feedback linearization of the weakly observable subsystem. Finally, a full characterization is derived for the nonlinear time delay system to have an unobservable subsystem not directly affected by the input and a weakly observable subsystem which is linearizable by feedback. The performed analysis is carried out within a new geometric framework recently introduced in the literature.
\end{abstract}

\section{Introduction}

In this chapter the conditions are investigated under which a given nonlinear single input- single output continuous time system, affected by constant commensurate delays, can be represented up to a feedback as the connection of two subsystems, a weakly observable subsystem, and an unobservable one. The latter naturally yields the notion of zero dynamics of the system. It is also investigated under which conditions, the weakly observable subsystem can be rendered linear. The interest in such a canonical form arises in various control problems including the study of stability and stabilization [14].

As well known, in the continuous delay-free case, under the assumption of defined relative degree, such a decomposition is always possible, and it is referred to as

\author{
C. Califano $(\otimes)$ \\ Dipartimento di Ingegneria Informatica Automatica e Gestionale "Antonio Ruberti", \\ Università di Roma La Sapienza, Via Ariosto 25, 00185 Rome, Italy \\ e-mail: califano@dis.uniroma1.it \\ C.H. Moog \\ L'UNAM, IRCCyN, UMR C.N.R.S. 6597, 1 rue de la Noë, BP 92101, \\ 44321 Nantes Cedex 3, France \\ e-mail: moog@ieee.org
}


normal form [10]. In this case the input-output behavior can always be rendered linear through an appropriate choice of the feedback law and can be represented as a chain of integrators. Furthermore for single output systems, it has also been shown that the unobservable dynamics is independent of the control, a property which is in general lost in the multi-output case. This property which has some interesting implications on the study of the zero dynamics of the system is not guaranteed for nonlinear discrete time single-input single-output systems [3]. In fact the assumption of defined relative degree allows to achieve the desired decomposition, but in general the unobservable subsystem is influenced by the control.

The case of single-input systems of retarded type is even more involved since the assumption of well defined relative degree does not guarantee anymore the existence of such a decomposition. Furthermore in order to get a linear input-output behavior some additional conditions are needed, and even if these conditions are satisfied, a representation of such a behavior as a chain of integrators can be achieved only under very strong conditions. It is also shown that the unobservable dynamics is in general influenced by the control. Some preliminary results were presented in [4].

The outline of this book chapter is as follows. The statements of the problems under investigation are given in Sect.2, while some recalls on the algebraic and geometric tools used in the chapter for dealing with nonlinear time-delay systems are given in Sect. 3. Section 4 is devoted to the main results. Some concluding remarks are given in Sect. 5 .

\section{Preliminaries and Problem Statements}

A class of nonlinear single-input single-output systems is considered whose state variables and control are subject to constant and commensurate delays. Without loss of generality, and after an eventual preliminary sampling (as explained in [9, 12]), the dynamics reads:

$$
\begin{aligned}
\Sigma: \dot{x}_{[0]} & =F\left(\mathbf{x}_{[s]}\right)+\sum_{j=0}^{s} G_{j}\left(\mathbf{x}_{[s]}\right) u_{[0]}(-j) \\
y_{[0]} & =H\left(\mathbf{x}_{[s]}\right)
\end{aligned}
$$

where

$$
\mathbf{x}_{[s]}^{T}=\left(x^{T}(t), \cdots x^{T}(t-s)\right) \in \mathbb{R}^{(s+1) n},
$$

represents the first $(s+1) n$ components of the state of the infinite dimensional system (1). The notations $x_{[0]}=x(t) \in \mathbb{R}^{n}, u_{[0]}=u(t) \in \mathbb{R}$ and $y_{[0]}=y(t) \in \mathbb{R}$ stand for the instantaneous values of the state, the input and the output. The functions $F\left(\mathbf{x}_{[s]}\right), G_{j}\left(\mathbf{x}_{[s]}\right)$ for $j \in[0, s]$ and $H\left(\mathbf{x}_{[s]}\right)$ are assumed analytic in their arguments. Additional assumptions such as dealing with local/global Lipschitz functions or 
forward completeness may be introduced depending on the requirements on the existence/uniqueness of the solution of (1). These assumptions play a key role when dealing with stability problems.

Denote by

$$
\mathbf{x}_{[s]}^{T}(-p)=\left(x^{T}(t-p), \cdots x^{T}(t-s-p)\right)
$$

in a similar vein, we define $\mathbf{u}_{[s]}$ and $\mathbf{u}_{[s]}(-p)$. When there is no confusion, the subscript will be dropped so that $\mathbf{x}$ represents $\mathbf{x}_{[s]}$ and $\mathbf{x}(-p)$ represents $\mathbf{x}_{[s]}(-p)$. On the contrary if a function $\tau(\cdot)$ depends not only on $\mathbf{x}_{[s]}$, but also on some future values of the variable $x(t)$, in order to put in evidence that such a function is not causal, we will say that $\tau(\cdot)=\tau\left(\mathbf{x}_{[*]}\right)$. Given a function $\tau\left(\mathbf{x}_{[s]}\right)$, its $r$ th derivative with respect to time will be denoted as

$$
\tau^{(r)}\left(\mathbf{x}_{[s]}\right):=\frac{d^{r} \tau\left(\mathbf{x}_{[s]}\right)}{d t^{r}}
$$

The notation $\mathbf{x}_{[\gamma]}^{0}$ represents an equilibrium point.

In the following, $\mathscr{K}$ will be used to denote the field of meromorphic functions of a finite number of variables in

$$
\left\{\mathbf{x}_{[0]}(-i), \mathbf{u}_{[0]}(-i), \cdots, \mathbf{u}_{[0]}^{(k)}(-i), k, i \in \mathbb{N}\right\}
$$

where $\mathbb{N}$ is the set of natural numbers. The notation " $d$ " will represent the differential operator, while " $\delta$ " will represent the backward time-shift operator: for $a\left(\mathbf{x}_{[\alpha]}\right), f\left(\mathbf{x}_{[\beta]}\right) \in \mathscr{K}:$

$$
\delta\left[a\left(\mathbf{x}_{[\alpha]}\right) d f\left(\mathbf{x}_{[\beta]}\right)\right]=a\left(\mathbf{x}_{[\alpha]}(-1)\right) \delta d f\left(\mathbf{x}_{[\beta]}\right)=a\left(\mathbf{x}_{[\alpha]}(-1)\right) d f\left(\mathbf{x}_{[\beta]}(-1)\right) .
$$

The left ring of polynomials in $\delta$ with coefficients in $\mathscr{K}$ is denoted $\mathscr{K}(\delta]$.

In this framework a one-form is written as

$$
\omega(\mathbf{x}, \delta) d x_{[0]}=\sum_{i=1}^{n} \omega_{i}(\mathbf{x}, \delta) d x_{i,[0]}, \omega_{i}(\mathbf{x}, \delta) \in \mathscr{K}(\delta], i \in[1, n] .
$$

If there exists $f(\mathbf{x})$ such that $d f(\mathbf{x})=\omega(\mathbf{x}, \delta) d x_{[0]}$, then the one-form $\omega(\mathbf{x}, \delta) d x_{[0]}$ is exact. A set of one-forms $\left\{\omega_{i}(\mathbf{x}, \delta) d x_{[0]}, i \in[1, j]\right\}$ are independent over $\mathscr{K}(\delta]$ if

$$
\sum_{i=1}^{j} \alpha_{i}(\mathbf{x}, \delta) \omega_{i}(\mathbf{x}, \delta)=0
$$

only if $\alpha_{i}(\mathbf{x}, \delta)=0, \forall i \in[1, j]$. Finally $\Delta=\operatorname{span}_{\mathscr{K}}\left\{r_{1}(\mathbf{x}), \cdots, r_{j}(\mathbf{x})\right\}$ denotes the distribution spanned by the $r_{i}(\mathbf{x})$ 's. Any element of $\Delta$ can be expressed as a linear 
combination of the $r_{i}(\mathbf{x})$ 's with coefficient in $\mathscr{K} . \bar{\Delta}$ will denote its involutive closure. Analogously $\Delta(\mathbf{x}, \delta)=\operatorname{span}_{\mathscr{K}(\delta)}\left\{r_{1}(\mathbf{x}, \delta), \cdots, r_{j}(\mathbf{x}, \delta)\right\}$ denotes the submodule spanned by the $r_{i}(\mathbf{x}, \delta)$ 's. Any element of $\Delta(\mathbf{x}, \delta)$ can be expressed as

$$
r(\mathbf{x}, \delta)=\sum_{i=0}^{j} r_{i}(\mathbf{x}) \alpha_{i}(\mathbf{x}, \delta)
$$

with $\alpha_{i}(\mathbf{x}, \delta) \in \mathscr{K}(\delta]$.

Before stating the different addressed problems, we will recall the notions of relative degree of a given output function, observability of the given system, bicausality of a change of coordinates and regular static state feedback, which play a key role. More precisely let us recall that the relative degree $r$ of the output $y_{[0]}$ is defined as the smallest integer $r$, such that the $j$ th derivative of the output function $y_{[0]}^{(j)}$ is not influenced by the control, for $0 \leq j \leq r-1$, while $y_{[0]}^{(r)}$ is influenced by the (eventually delayed) control. For the class of system considered, algebraic conditions are given in Definition 6, which characterize the relative degree through the use of the extended Lie derivative (Definition 5).

Definition 1 (Observability, [11]) System $\Sigma$ with instantaneous state variable $x_{[0]} \in$ $\mathbb{R}^{n}$ is said to be weakly observable if the observability matrix $\mathbf{O}(\mathbf{x}, \delta)$ satisfying

$$
\left(\begin{array}{c}
d y_{[0]} \\
\vdots \\
d y_{[0]}^{(n-1)}
\end{array}\right)=\mathbf{O}(\mathbf{x}, \delta) d x_{[0]},
$$

is a full rank matrix.The system is strongly observable if $\mathbf{O}(\mathbf{x}, \delta)$ is unimodular, i.e. it admits a polynomial inverse.

Since we will study the equivalence of systems linked through a change of coordinates, recall the following definition of bicausal change of coordinates adapted from [15].

Definition 2 (Bicausal change of coordinates) Consider system $\Sigma$ in the state coordinates $x$. The mapping $z_{[0]}=\varphi\left(\mathbf{x}_{[\alpha]}\right)$, where $\alpha \in \mathbb{N}$ and $\varphi \in \mathscr{K}^{n}$, is a local bicausal change of coordinates for $\Sigma$ if there exists an integer $\ell \in \mathbb{N}$ and a function $\psi\left(\mathbf{z}_{[\ell]}\right) \in \mathscr{K}^{n}$ such that, assuming $z_{[0]}$ and $x_{[0]}$ defined for $t \geq-(\alpha+\ell)$, then $\psi\left(\varphi\left(\mathbf{x}_{[\alpha]}\right), \cdots, \varphi\left(\mathbf{x}_{[\alpha]}(-\ell)\right)=x_{[0]}\right.$ for $t \geq 0$. 
Definition 3 (Bicausal regular static state feedback) Consider the input $w_{[0]} \in \mathbf{R}^{m}$. The feedback

$$
w_{[0]}=\alpha(\mathbf{x})+\sum_{j=0}^{i} \beta_{j}(\mathbf{x}) v_{[0]}(-j)
$$

is a local bicausal regular static state feedback if there exists an integer $\ell \in \mathbb{N}$ and functions $\bar{\alpha}(\mathbf{x})$ and $\bar{\beta}_{\mu}(\mathbf{x})$ such that,

$$
\alpha(\mathbf{x})+\sum_{j=0}^{i} \beta_{j}(\mathbf{x})\left(\bar{\alpha}(\mathbf{x}(-j))+\sum_{\mu=0}^{\ell} \bar{\beta}_{\mu}(\mathbf{x}(-j)) w_{[0]}(-\mu-j)\right)=w_{[0]} .
$$

According to the definition, the regularity and bicausality of the feedback is equivalent to the unimodularity of the matrix

$$
\beta(\mathbf{x}, \delta)=\sum_{j=0}^{i} \beta_{j}(\mathbf{x}) \delta^{j}
$$

In the single input case, considered in the present chapter, it thus reduces to the independence of $\delta$, that is $\beta(\mathbf{x}, \delta)=\beta(\mathbf{x}) \neq 0$, or equivalently $\beta_{0}(\mathbf{x}) \neq 0$ and $\beta_{j}(\mathbf{x})=0$ for all $j \in[1, i]$.

The following problems can now be set.

Problem Statement 1. [Existence of the Generalized Normal Form] Given system (1), with output $y_{[0]}$ characterized by the well defined relative degree $r$, find, if possible, a regular bicausal static state feedback

$$
u_{[0]}=\alpha(\mathbf{x})+\beta(\mathbf{x}) v_{[0]},
$$

and a bicausal change of coordinates

$$
z_{[0]}=\left(\begin{array}{c}
z_{1,[0]} \\
z_{2,[0]}
\end{array}\right)=\varphi(\mathbf{x})
$$

with $z_{1,[0]} \in \mathbb{R}^{r}$, such that in the new coordinates the closed-loop system reads as:

$$
\begin{aligned}
& \dot{z}_{1,[0]}=\theta_{1}\left(\mathbf{z}_{1,[\ell]}\right)+\sum_{j=0}^{\ell} \theta_{2 j}\left(\mathbf{z}_{1,[\ell]}\right) v_{[0]}(-j), \\
& \dot{z}_{2,[0]}=\eta_{1}\left(\mathbf{z}_{[\ell]}\right)+\sum_{j=0}^{\ell} \eta_{2 j}\left(\mathbf{z}_{[\ell]}\right) v_{[0]}(-j), \quad y_{[0]}=\tilde{H}\left(\mathbf{z}_{1,[\ell]}\right),
\end{aligned}
$$

where the subsystem 


$$
\dot{z}_{1,[0]}=\theta_{1}\left(\mathbf{z}_{1,[\ell]}\right)+\sum_{j=0}^{\ell} \theta_{2 j}\left(\mathbf{z}_{1,[\ell]}\right) v_{[0]}(-j), \quad y_{[0]}=\tilde{H}\left(\mathbf{z}_{1,[\ell]}\right)
$$

is weakly observable.

Such a problem can be further developed by requiring that the input-output behavior is described by a linear system, which leads to the second problem examined in this chapter:

Problem Statement 2. [Existence of the Normal Form] Given system (1), with output $y_{[0]}$ characterized by the well defined relative degree $r$, find, if possible, a regular bicausal static state feedback $u_{[0]}=\alpha(\mathbf{x})+\beta(\mathbf{x}) v_{[0]}$, and a bicausal change of coordinates

$$
z_{[0]}=\left(\begin{array}{l}
z_{1,[0]} \\
z_{2,[0]}
\end{array}\right)=\varphi(\mathbf{x})
$$

with $z_{1,[0]} \in \mathbb{R}^{r}$, such that in the new coordinates the closed-loop system reads as (3) with a linear (weakly) observable input-output behavior, that is with

$$
\begin{aligned}
\dot{z}_{1,[0]} & =\theta_{1}\left(\mathbf{z}_{1,[\ell]}\right)+\sum_{j=0}^{\ell} \theta_{2 j}\left(\mathbf{z}_{1,[\ell]}\right) v_{[0]}(-j)=\sum_{j=0}^{\ell} A_{j} z_{1,[0]}(-j)+\sum_{j=0}^{\ell} B_{j} v_{[0]}(-j), \\
y_{[0]} & =\tilde{H}\left(\mathbf{z}_{1,[\ell]}\right)=\sum_{j=0}^{\ell} C_{j} z_{1,[0]}(-j),
\end{aligned}
$$

where the polynomial matrices

$$
A(\delta)=\sum_{j=0}^{\ell} A_{j} \delta^{j}, B(\delta)=\sum_{j=0}^{\ell} B_{j} \delta^{j}, \text { and } C(\delta)=\sum_{j=0}^{\ell} C_{j} \delta^{j}
$$

define a linear (weakly) observable system.

$\triangleleft$

Finally, the conditions under which the unobservable dynamics defined by the derivative of $z_{2,[0]}$ in (3) is not affected by the control $v$ are investigated; that is the bicausal change of coordinates

$$
z_{[0]}=\left(\begin{array}{l}
z_{1,[0]} \\
z_{2,[0]}
\end{array}\right)=\varphi(\mathbf{x})
$$

can be chosen to guarantee that

$$
\dot{z}_{2,[0]}=\eta_{1}\left(\mathbf{z}_{[\ell]}\right) .
$$


This has interesting consequences when studying the stability properties of the whole system as discussed in Sect. 5.

Remark 1 System (1) is defined for $t \geq 0$ once the initial condition $x(t)=\varphi(t)$ is set on the interval $[-s, 0]$. For the equivalence to the form (3) to hold true for $t \geq 0$, it may be necessary to consider the initial condition $x(t)=\varphi(t)$ set on a greater interval $[-\bar{s}, 0]$, to guarantee that the change of coordinates and the static state feedback are well defined for $t \geq 0$, as well as the initial condition $z(t)=\bar{\varphi}(t)$ which must be well defined on the interval $[-\ell, 0]$.

This study is performed within a new geometric framework which was recently introduced in [1], and appeared to be successful to derive various canonical forms $[1,2]$. More precisely, the conditions are stated in terms of extended Lie brackets of vector fields which are defined on appropriate finite dimensional manifolds.

\section{Recalling Results on Algebraic and Geometric Tools}

This Section is devoted to recall some basic tools and results which will be used to address the problems stated in Sect.2. The approach has shown to be efficient for solving various problems for nonlinear time delay systems, including the identification of the delay [16].

\subsection{Differential Representation}

Given system $\Sigma$ defined by (1), its differential representation is

$$
\begin{aligned}
\Sigma_{L}: & d \dot{x}_{[0]}=f\left(\mathbf{x}_{[s]}, \mathbf{u}_{[s]}, \delta\right) d x_{[0]}+g_{1}\left(\mathbf{x}_{[s]}, \delta\right) d u_{[0]} \\
& d y_{[0]}=h\left(\mathbf{x}_{[s]}, \delta\right) d x_{[0]}
\end{aligned}
$$

with

$$
\begin{aligned}
f\left(\mathbf{x}_{[s]}, \mathbf{u}_{[s]}, \delta\right) & =\sum_{i=0}^{s} \frac{\partial F\left(\mathbf{x}_{[s]}\right)}{\partial x_{[0]}(-i)} \delta^{i}+\sum_{j=0}^{s} u_{[0]}(-j) \sum_{i=0}^{s} \frac{\partial G_{j}\left(\mathbf{x}_{[s]}\right)}{\partial x_{[0]}(-i)} \delta^{i} \\
g_{1}\left(\mathbf{x}_{[s]}, \delta\right) & =\sum_{j=0}^{s} G_{j}\left(\mathbf{x}_{[s]}\right) \delta^{j}=\sum_{j=0}^{s} g_{1}^{j}\left(\mathbf{x}_{[s]}\right) \delta^{j} \\
h\left(\mathbf{x}_{[s]}, \delta\right) & =\sum_{j=0}^{s} \frac{\partial H\left(\mathbf{x}_{[s]}\right)}{\partial x_{[0]}(-j)} \delta^{j}
\end{aligned}
$$




\subsection{Left- and Right-Annihilators}

- Let $T(\mathbf{x}, \delta)$ be a $(n \times r)$ polynomial matrix of rank $r$. Its left-annihilator $\Omega(\mathbf{x}, \delta)$ consists of all row vectors $\omega(\mathbf{x}, \delta)$ such that $\omega(\mathbf{x}, \delta) T(\mathbf{x}, \delta)=0$. By definition, the submodule $\Omega(\mathbf{x}, \delta)$ is closed in the following sense of left-closure:

$$
P(\mathbf{x}, \delta) \pi \in \Omega \Rightarrow \pi \in \Omega
$$

where $P(\mathbf{x}, \delta)$ stands for a polynomial operator.

- Let $T(\mathbf{x}, \delta)$ be a $(r \times n)$ polynomial matrix of rank $r$. Its right-annihilator $\Delta\left(\mathbf{x}_{[*]}, \delta\right)$ consists of all column vectors $v\left(\mathbf{x}_{[*]}, \delta\right)$ such that $T(\mathbf{x}, \delta) v\left(\mathbf{x}_{[*]}, \delta\right)=0$. By definition, the right-kernel is closed in the following sense of right-closure:

$$
v\left(\mathbf{x}_{[*]}, \delta\right) P\left(\mathbf{x}_{[*]}, \delta\right) \in \Delta\left(\mathbf{x}_{[*]}, \delta\right) \Rightarrow v\left(\mathbf{x}_{[*]}, \delta\right) \in \Delta\left(\mathbf{x}_{[*]}, \delta\right)
$$

- The left closure of the polynomial matrix $T(\mathbf{x}, \delta)$ is obtained computing the leftannihilator of the right-annihilator of $T(\mathbf{x}, \delta)$.

- The right closure of the polynomial matrix $T(\mathbf{x}, \delta)$ is obtained computing the right-annihilator of the left-annihilator of $T(\mathbf{x}, \delta)$.

- Note that starting from the causal $(n \times r)$ polynomial matrix $T(\mathbf{x}, \delta)$ of rank $r$, its left-annihilator can always be expressed by causal generators. On the contrary starting from the causal $(r \times n)$ polynomial matrix $T(\mathbf{x}, \delta)$ of rank $r$, it may not be possible to express its right-annihilator through causal generators, that is the entries of the right-annihilator are polynomials in $\delta$ whose coefficients are functions which may depend on future time instants $t+k$ for some $k>0$. For instance, consider

$$
T(\mathbf{x}, \delta)=\left(\begin{array}{c}
x_{1}(-1)+x_{1} \delta \\
x_{2} \delta
\end{array}\right)
$$

Then its left annihilator is

$$
\omega=\left(x_{2} \delta,-x_{1}(-2)-x_{2} \frac{x_{1}(-1)}{x_{2}(-1)} \delta\right),
$$

which is causal. Now consider the transpose of $T(\mathbf{x}, \delta)$, namely,

$$
\bar{T}(\mathbf{x}, \delta)=\left(x_{1}(-1)+x_{1} \delta, x_{2} \delta\right) .
$$

Its right annihilator is

$$
v=\left(\begin{array}{c}
x_{2} \delta \\
-x_{1}-\frac{x_{1}(+1)}{x_{2}(+1)} x_{2} \delta
\end{array}\right),
$$

which is not causal. 


\subsection{The Action of a Bicausal Change of Coordinates}

Given the bicausal change of coordinates $z_{[0]}=\varphi(\mathbf{x})$, let us consider the associated differential representation $d \mathbf{z}_{[0]}=T(\mathbf{x}, \delta) d x_{[0]}$. Then, $T\left(\mathbf{x}_{[\alpha]}, \delta\right)$ is unimodular. Moreover, its inverse has a polynomial degree $\gamma \leq \alpha(n-1)$. Moreover, under such a bicausal change of coordinates, the differential representation (4) is transformed into

$$
d \dot{z}_{[0]}=\tilde{f}(\mathbf{z}, \mathbf{u}, \delta) d z_{[0]}+\tilde{g}_{1}(\mathbf{z}, \delta) d u_{[0]}, \quad d y_{[0]}=\tilde{h}(\mathbf{z}, \delta) d z_{[0]},
$$

where

$$
\begin{aligned}
& \tilde{f}(\mathbf{z}, \mathbf{u}, \delta)=\left[(T(\mathbf{x}, \delta) f(\mathbf{x}, \mathbf{u}, \delta)+\dot{T}(\mathbf{x}, \delta)) T^{-1}(\mathbf{x}, \delta)\right]_{\varphi^{-1}(\mathbf{z})} \\
& \tilde{g}_{1}(\mathbf{z}, \delta)=\left(T(\mathbf{x}, \delta) g_{1}(\mathbf{x}, \delta)\right)_{\varphi^{-1}(\mathbf{z})}
\end{aligned}
$$

Remark 2 The rank of $T(\mathbf{x}, \delta)$ may drop around some singular function $\varphi(\mathbf{x})$, which may eventually be a trajectory for the system. In this case the equivalence does not hold true around such a singular trajectory.

\subsection{Extended Lie Bracket}

We next recall the definition of the extended Lie bracket introduced in [1] to tackle nonlinear time-delay systems and which is used to characterize the integrability of 1 -forms defined not only from state variables at time $t$ but also from their time shifts.

Definition 4 (Extended Lie Bracket) Let

$$
r_{\beta}(\mathbf{x}, \delta)=\sum_{j=0}^{s} r_{\beta}^{j}(\mathbf{x}) \delta^{j}
$$

where $\beta=1,2$. The extended Lie bracket

$$
\left[r_{1}^{k}(\mathbf{x}), r_{2}^{l}(\mathbf{x})\right]_{E_{i}}
$$

is defined on $\mathbb{R}^{(i+1) n}, i \geq 0$, by

$$
\left[r_{1}^{k}(\cdot), r_{2}^{l}(\cdot)\right]_{E_{i}}=\sum_{j=0}^{\bar{k}}\left(\left[r_{1}^{k-j}(\cdot), r_{2}^{l-j}(\cdot)\right]_{E_{0}}\right)_{\mid(\mathbf{x}(-j), \mathbf{u}(-j))}^{T} \frac{\partial}{\partial x_{[0]}(-j)}
$$


with $\bar{k}=\min (k, l, i)$, and

$$
\left[r_{1}^{k}(\cdot), r_{2}^{l}(\cdot)\right]_{E_{0}}=\sum_{i=0}^{k} \frac{\partial r_{2}^{l}(\mathbf{x})}{\partial x_{[0]}(-i)} r_{1}^{k-i}(\mathbf{x}(-i))-\sum_{i=0}^{l} \frac{\partial r_{1}^{k}(\mathbf{x})}{\partial x_{[0]}(-i)} r_{2}^{l-i}(\mathbf{x}(-i))
$$

The extended Lie bracket makes sense for the infinite dimensional system

$$
\begin{gathered}
\dot{x}_{[0]}=F\left(\mathbf{x}_{[s]}\right)+\sum_{j=0}^{s} G_{j}\left(\mathbf{x}_{[s]}\right) u_{[0]}(-j) \\
\dot{x}_{[0]}(-1)=F\left(\mathbf{x}_{[s]}(-1)\right)+\sum_{j=0}^{s} G_{j}\left(\mathbf{x}_{[s]}(-1)\right) u_{[0]}(-j-1)
\end{gathered}
$$

which is associated to the time-delay system (1). The computation of the extended Lie bracket can be implemented in some computer algebra software as done in [6].

As for delay-free systems, it is useful to introduce an extended Lie derivative whose definition is given below and differs slightly from the one in $[7,13]$.

Definition 5 (Extended Lie Derivative) Given a function $\lambda\left(\mathbf{x}_{[s]}\right)$ and the vector

$$
r_{i}(\mathbf{x}, \delta)=\sum_{j=0}^{\bar{s}} r_{i}^{j}(\mathbf{x}) \delta^{j}
$$

the extended Lie derivative $L_{r_{i}^{j}(\mathbf{x})} \lambda\left(\mathbf{x}_{[s]}\right)$ of $\lambda\left(\mathbf{x}_{[s]}\right)$ in the direction $r_{i}^{j}$ is

$$
L_{r_{i}^{j}(\mathbf{x})} \lambda\left(\mathbf{x}_{[s]}\right)=\sum_{l=0}^{j} \frac{\partial \lambda\left(\mathbf{x}_{[s]}\right)}{\partial x_{[0]}(-l)} r_{i}^{j-l}(\mathbf{x}(-l))
$$

One thus gets

$$
\left[r_{1}^{k}(\cdot), r_{2}^{l}(\cdot)\right]_{E_{0}}=\left(L_{r_{1}^{k}(\mathbf{x})} r_{2}^{l}(\mathbf{x})-L_{r_{2}^{l}(\mathbf{x})} r_{1}^{k}(\mathbf{x})\right)^{T} \frac{\partial}{\partial x_{[0]}}
$$

This allows to recover standard definitions of Lie derivatives and Lie brackets which are used in the delay free case. From (1), consider now the polynomial vector

$$
\mathbf{F}\left(\mathbf{x}_{[s]}, \delta\right)=\sum_{j=0}^{n s} F^{j}\left(\mathbf{x}_{[s]}\right) \delta^{j}=\sum_{j=0}^{n s} F\left(\mathbf{x}_{[s]}\right) \delta^{j} .
$$

The derivative of $\lambda\left(\mathbf{x}_{[s]}\right)$ of order $i$ which is computed for $\mathbf{u}=0$, is given by 


$$
\lambda^{(i)}(\mathbf{x}, 0)=L_{F^{n s}(\mathbf{x})}^{i} \lambda\left(\mathbf{x}_{[s]}\right),
$$

for $i \in[1, n]$. The definition of relative degree can then be stated as follows.

Definition 6 The function $\lambda\left(\mathbf{x}_{[s]}\right)$ has relative degree $k>0$ if

$$
L_{g_{1}^{j}} L_{F^{n s}}^{i} \lambda(\mathbf{x}) \equiv 0 \quad \forall j \geq 0, \forall 0 \leq i<k-1,
$$

and if there exists an integer $j \geq 0$ such that

$$
L_{g_{1}^{j}} L_{F^{n s}}^{k-1} \lambda(\mathbf{x}) \neq 0
$$

The relative degree is said to be strong if (11) is fulfilled for $j=0$.

\subsection{Integrability}

Next, let us recall a fundamental result on integrability of a submodule, giving conditions under which the left annihilator of the submodule is spanned by exact one-forms. The following is a more elegant revisited version of a result in [1]. Consider

$$
P_{j}(\mathbf{x}, \delta)=\left[r_{1}(\mathbf{x}, \delta), \cdots, r_{j}(\mathbf{x}, \delta)\right]=\sum_{l=0}^{s} P_{j l}(\mathbf{x}) \delta^{l}
$$

where $P_{j 0}(\mathbf{x})$ has full column rank $j$ and

$$
r_{k}(\mathbf{x}, \delta)=\sum_{l=0}^{s} r_{k}^{l}(\mathbf{x}) \delta^{l}, k \in[1, j],
$$

and let $s$ be the maximum delay in the $\mathbf{x}$ variable. Denoting, with some abuse of notations, in compact form

$$
r^{l}=r_{1}^{l}, \cdots, r_{j}^{l}
$$

and by $I_{n}$ the $n \times n$ identity matrix, consider the distributions $\Delta_{i}, i \geq 0$ which are defined on $\mathbb{R}^{(i+s+1) n}$ as follows:

$$
\Delta_{0}=\operatorname{span}_{\mathscr{K}}\left\{\begin{array}{ccccc}
r^{0} & \cdots & r^{s} & 0 & \ldots \\
\cdots & I_{n} & & \\
\cdots & \ddots & \ddots & \\
\cdots & & 0 & I_{n}
\end{array}\right\}
$$




$$
\begin{aligned}
& \Delta_{1}=\operatorname{span}_{\mathscr{K}}\left\{\begin{array}{ccccccc}
r^{0} & \cdots & r^{s} & 0 & \cdots & \\
0 & r^{0}(-1) & \cdots & r^{s}(-1) & 0 & \cdots & \\
& & & & & & \\
& & & & & & \\
& & \cdots & & & \ddots & \\
& & \cdots & & & 0 & I_{n}
\end{array}\right\}
\end{aligned}
$$



Let $\rho_{i}=\operatorname{dim}\left(\bar{\Delta}_{i}\right)$ in the neighborhood of $\mathbf{x}^{0}$.

Theorem 1 Consider the submodule

$$
\Delta(\mathbf{x}, \delta)=\operatorname{span}_{\mathscr{K}(\delta]}\left\{r_{1}(\mathbf{x}, \delta), \cdots, r_{j}(\mathbf{x}, \delta)\right\}, \text { where } r_{i}(\mathbf{x}, \delta)=\sum_{l=0}^{s} r_{i}^{l}\left(\mathbf{x}_{[s]}\right) \delta^{l},
$$

and such that the matrices

$$
P_{j}(\mathbf{x}, \delta)=\left(r_{1}(\mathbf{x}, \delta), \cdots, r_{j}(\mathbf{x}, \delta)\right)=\sum_{l=0}^{s} P_{j l}(\mathbf{x}) \delta^{l}
$$

and $P_{j 0}(\mathbf{x})$ have rank $j$. Let $\Delta_{i}$ be the set of distributions defined by (12), which have by assumption constant dimension $\rho_{i}=\operatorname{dim}\left(\bar{\Delta}_{i}\right)$ locally around $\mathbf{x}_{[i]}^{0}$, with $\rho_{-1}=n s$ by definition. Then $\Delta(\mathbf{x}, \delta)$ is completely integrable if and only if there exists an integer $\gamma$ such that $\rho_{\gamma}-\rho_{\gamma-1}=j$.

If the given submodule $\Delta(\mathbf{x}, \delta)$ is not completely integrable, one may wish to compute the largest number of exact one-forms independent over $\mathscr{K}(\delta]$ which are in the left-kernel of $\Delta(\mathbf{x}, \delta)$. The following result holds:

Theorem 2 Consider the submodule

$$
\Delta(\mathbf{x}, \delta)=\operatorname{span}_{\mathscr{K}(\delta]}\left\{r_{1}(\mathbf{x}, \delta), \cdots, r_{j}(\mathbf{x}, \delta)\right\}
$$

with

$$
r_{i}(\mathbf{x}, \delta)=\sum_{l=0}^{s} r_{i}^{l}\left(\mathbf{x}_{[s]}\right) \delta^{l}
$$


and such that matrices

$$
P_{j}(\mathbf{x}, \delta)=\left(r_{1}(\mathbf{x}, \delta), \cdots, r_{j}(\mathbf{x}, \delta)\right)=\sum_{l=0}^{s} P_{j l}(\mathbf{x}) \delta^{l}
$$

and $P_{j 0}(\mathbf{x})$ have rank $j$. Let $\Delta_{i}$ be the set of distributions defined by (12), which have by assumption constant dimension $\rho_{i}=\operatorname{dim}\left(\bar{\Delta}_{i}\right)$ locally around $\mathbf{x}_{[i]}^{0}$, where $\rho_{-1}=n$ s by definition. Let $\gamma$ be the smallest index such that $\rho_{\gamma+k}-\rho_{\gamma+k-1}=\ell \geq j$, $\forall k \geq 0$. The smallest completely integrable submodule $\bar{\Delta}(\mathbf{x}, \delta) \supset \Delta(\mathbf{x}, \delta)$ has rank $\ell$. Consequently, there exist $n-\ell$ exact one-forms independent over $\mathscr{K}(\delta]$ which generate the left kernel of $\Delta(\mathbf{x}, \delta)$.

We end this section by stating the following result from [5], which is fundamental for developing the results in the next section.

Theorem 3 Let $\Delta(\mathbf{x}, \delta)$ be a given submodule and let $\bar{\Delta}(\mathbf{x}, \delta)$ denote the smallest completely integrable submodule such that $\bar{\Delta}(\mathbf{x}, \delta) \supset \Delta(\mathbf{x}, \delta)$. Let $j$ be the rank of $\bar{\Delta}(\mathbf{x}, \delta)$, then there exist $n-j$ exact one forms $\left(d \lambda_{1}, \cdots, d \lambda_{n-j}\right)$ which generate the left annihilator of $\bar{\Delta}(\mathbf{x}, \delta)$. Furthermore there exist $j$ exact one forms $\left(d \lambda_{n-j+1}, \cdots, d \lambda_{n}\right)$, which can be taken to define a basis completion, that is

$$
\left(\begin{array}{c}
d \lambda_{1} \\
\vdots \\
d \lambda_{n}
\end{array}\right)=T(\mathbf{x}, \delta) d x_{[0]}
$$

with $T(\mathbf{x}, \delta)$ unimodular.

\section{Main Results}

In this section we address the problem of characterizing the different geometrical structures analyzed in the Introduction. The conditions are linked to two different properties of the system, basically, what can be achieved under bicausal change of coordinates, and what can be achieved under regular bicausal static state feedback.

\subsection{Generalized Normal Form}

Hereafter we analyze the conditions under which there exist a bicausal change of coordinates and a regular bicausal static state feedback thanks to which the closedloop system can be decomposed in the generalized normal form 


$$
\begin{aligned}
\dot{z}_{1,[0]} & =\theta_{1}\left(\mathbf{z}_{1,[\ell]}\right)+\sum_{j=0}^{\ell} \theta_{2 j}\left(\mathbf{z}_{1,[\ell]}\right) v_{[0]}(-j), \\
\dot{z}_{2,[0]} & =\eta_{1}\left(\mathbf{z}_{[\ell]}\right)+\sum_{j=0}^{\ell} \eta_{2 j}\left(\mathbf{z}_{[\ell]}\right) v_{[0]}(-j) \\
y_{[0]} & =\tilde{H}\left(\mathbf{z}_{1,[\ell]}\right),
\end{aligned}
$$

with $z_{1,[0]} \in \mathbb{R}^{r}, r$ being the relative degree of the output.

Before enouncing the necessary and sufficient conditions, the following result is required, which enlightens under which conditions it is possible to define a bicausal change of coordinates such that the output function and its derivative up to the order $r-1$ can be expressed as functions of the variable $z_{1,[0]} \in \mathbb{R}^{r}$ and its delayed values. This condition, which is necessary to solve Problem 1, does not follow from the assumption of defined relative degree, as it happens in the delay-free case.

The following result holds:

Lemma 1 Given system (1), with output $y_{[0]}$ characterized by the well defined relative degree $r$, there exists a bicausal change of coordinates

$$
z_{[0]}=\left(\begin{array}{l}
z_{1,[0]} \\
z_{2,[0]}
\end{array}\right)=\left(\begin{array}{l}
\varphi_{1}(\mathbf{x}) \\
\varphi_{2}(\mathbf{x})
\end{array}\right)=\varphi(\mathbf{x})
$$

with $z_{1,[0]} \in \mathbb{R}^{r}$, such that in the new coordinates

$$
d y_{[0]}^{(i)}=\varphi_{i+1}\left(\mathbf{z}_{1}\right) \quad i \in[0, r-1]
$$

if and only if setting

$$
\left(\begin{array}{c}
d y_{[0]} \\
\vdots \\
d y_{[0]}^{(r-1)}
\end{array}\right)=\mathbf{Y}(\mathbf{x}, \delta) d x_{[0]}
$$

the right annihilator $\Delta(\cdot, \delta)$ of $\mathbf{Y}(\mathbf{x}, \delta)$ is causal.

Proof Necessity. Assume for instance that there exists a bicausal change of coordinates

$$
z_{[0]}=\left(\begin{array}{l}
z_{1,[0]} \\
z_{2,[0]}
\end{array}\right)=\left(\begin{array}{l}
\varphi_{1}(\mathbf{x}) \\
\varphi_{2}(\mathbf{x})
\end{array}\right)=\varphi(\mathbf{x})
$$

with $z_{1,[0]} \in \mathbb{R}^{r}$, such that in the new coordinates the output and its derivative up to the order $r-1$ are expressed as functions of the variable $\mathbf{z}_{1}$ only. Let 


$$
d z_{[0]}=\left(\begin{array}{l}
d z_{1,[0]} \\
d z_{2,[0]}
\end{array}\right)=\left(\begin{array}{l}
T_{1}(\mathbf{x}, \delta) \\
T_{2}(\mathbf{x}, \delta)
\end{array}\right) d x_{[0]}
$$

Then one gets immediately that

$$
\left(\begin{array}{c}
d y_{[0]} \\
\vdots \\
d y_{[0]}^{(r-1)}
\end{array}\right)=\Psi\left(\mathbf{z}_{1}, \delta\right) d z_{1,[0]}=\Psi\left(\mathbf{z}_{1}, \delta\right)_{z_{1,[0]}=\varphi_{1}(\mathbf{x})} T_{1}(\mathbf{x}, \delta) d x_{[0]}=\mathbf{Y}(\mathbf{x}, \delta) d x_{[0]}
$$

Consider now the matrix $T^{-1}(\mathbf{x}, \delta)$. Since $T(\mathbf{x}, \delta)$ is unimodular by assumption, $T^{-1}(\mathbf{x}, \delta)$ is causal. Furthermore setting $T^{-1}(\mathbf{x}, \delta)=\left(L_{1}(\mathbf{x}, \delta), L_{2}(\mathbf{x}, \delta)\right)$, by construction $T_{1}(\mathbf{x}, \delta) L_{2}(\mathbf{x}, \delta)=0$. Consequently, setting $\bar{\Psi}(\mathbf{x}, \delta)=\Psi\left(\mathbf{z}_{1}, \delta\right)_{z_{1,[0]}=\varphi_{1}(\mathbf{x})}$,

$$
\mathbf{Y}(\mathbf{x}, \delta) L_{2}(\mathbf{x}, \delta)=\bar{\Psi}(\mathbf{x}, \delta) T_{1}(\mathbf{x}, \delta) L_{2}(\mathbf{x}, \delta)=0
$$

It follows that the right annihilator $\Delta(\cdot, \delta)$ of $\mathbf{Y}(\mathbf{x}, \delta)$ is given by $\Delta(\cdot, \delta)=L_{2}(\mathbf{x}, \delta)$, has rank $n-r$ and is causal.

Sufficiency. By assumption $\Delta(\cdot, \delta)$ is causal, has rank $n-r$ and there exist $r$ exact differentials, independent over $\mathscr{K}(\delta]$ which lay in the left annihilator. Consequently the rank of the involutive closure $\bar{\Delta}(\mathbf{x}, \delta)$ of $\Delta(\mathbf{x}, \delta)$ is still $n-r$ and there exists $r$ exact differentials $d \lambda_{1}(\mathbf{x}), \cdots, d \lambda_{r}(\mathbf{x})$ which generate the left annihilator, so that for $i \in[0, r-1]$,

$$
d y_{[0]}^{(i)}=\sum_{j=1}^{r} \alpha_{i j}(\mathbf{x}, \delta) d \lambda_{j}(\mathbf{x})
$$

Due to Theorem 3 it is also possible to compute $n-r$ functions $\lambda_{r+1}(\mathbf{x}), \cdots, \lambda_{n}(\mathbf{x})$, so that

$$
\left(\begin{array}{c}
d \lambda_{1}(\mathbf{x}) \\
\vdots \\
d \lambda_{n}(\mathbf{x})
\end{array}\right)=T(\mathbf{x}, \delta) d x_{[0]}
$$

with $T(\mathbf{x}, \delta)$ unimodular. As a consequence, the transformation

$$
z_{[0]}=\left(\begin{array}{c}
\lambda_{1}(\mathbf{x}) \\
\vdots \\
\lambda_{n}(\mathbf{x})
\end{array}\right)
$$

defines a bicausal change of coordinates. Denoting by $z_{1,[0]}$ the first $r$-components, and by $z_{2,[0]}$ the last $n-r$ components of $z_{[0]}$, in these new coordinates, we have that, due to Eq. (15), for $i \in[0, r-1]$ 


$$
d y_{[0]}^{(i)}=\sum_{j=1}^{n} \alpha_{i j}(\mathbf{x}, \delta) d \lambda_{j}(\mathbf{x})=\sum_{j=1}^{r} \alpha_{i j}(\mathbf{x}, \delta) d \lambda_{j}(\mathbf{x})=\sum_{j=1}^{r} \bar{\alpha}_{i j}(\mathbf{z}, \delta) d z_{1,[0]}
$$

Since on the left hand-side one has exact differentials, then necessarily $\bar{\alpha}_{i j}(\mathbf{z}, \delta)=$ $\bar{\alpha}_{i j}\left(\mathbf{z}_{1}, \delta\right)$, which ends the proof.

Theorem 4 Given system (1), with output $y_{[0]}$ characterized by defined relative degree $r$, there exist a regular bicausal static state feedback

$$
u_{[0]}=\hat{\alpha}(\mathbf{x})+\hat{\beta}(\mathbf{x}) v_{[0]},
$$

and a bicausal change of coordinates

$$
z_{[0]}=\left(\begin{array}{l}
z_{1,[0]} \\
z_{2,[0]}
\end{array}\right)=\varphi(\mathbf{x})
$$

with $z_{1,[0]} \in \mathbb{R}^{r}$, such that in the new coordinates the closed-loop system reads as the Generalized Normal Form (13), if and only if the following conditions hold:

(i) Let

$$
\left(\begin{array}{c}
d y_{[0]} \\
\vdots \\
d y_{[0]}^{(r-1)}
\end{array}\right)=\mathbf{Y}(\mathbf{x}, \delta) d x_{[0]}
$$

The right annihilator $\Delta(\cdot, \delta)$ of $\mathbf{Y}(\mathbf{x}, \delta)$ has rank $n-r$ and is causal.

(ii) $d y_{[0]}^{(r)}$, the differential of the rth derivative of the output $y^{(r)}\left(\mathbf{x}_{[s]}, \mathbf{u}_{[s]}\right)$, given by

$$
d y_{[0]}^{(r)}=a(\mathbf{x}, \mathbf{u}, \delta) d x_{[0]}+b(\mathbf{x}, \delta) d u_{[0]},
$$

satisfies the following properties:

(iia) $b(\mathbf{x}, \delta)$ in (17) can be factorized as

$$
b(\mathbf{x}, \delta)=\tilde{b}(\mathbf{x}, \delta) \beta(\mathbf{x})
$$

with $\tilde{b}(\mathbf{x}, \delta)=\sum_{j=0}^{\ell} \tilde{b}_{j}(\mathbf{x}) \delta^{\ell}$ such that

$$
d \tilde{b}_{j}(\mathbf{x}) \in \Delta(\mathbf{x}, \delta)^{\perp}, \quad \forall j \in[0, \ell]
$$

(iib) there exists a function $\alpha(\mathbf{x})$ such that denoting by

$$
\gamma(\mathbf{x}, \delta) d x_{[0]}=d \alpha(\mathbf{x})
$$

then, $a(\mathbf{x}, \mathbf{u}, \delta)$ in (17) computed for $\mathbf{u}=0$ and $\tilde{b}(\mathbf{x}, \delta)$ in (18) satisfy 


$$
(a(\mathbf{x}, 0, \delta)-\tilde{b}(\mathbf{x}, \delta) \gamma(\mathbf{x}, \delta)) d x_{[0]} \in \Delta(\mathbf{x}, \delta)^{\perp}
$$

If conditions $(i)$ and (ii) are satisfied, the coordinates $z_{[0]}=\varphi(\mathbf{x})$, which transform the closed-loop system in the form (13), can be chosen in such a way that $\theta_{2 j}\left(\mathbf{z}_{1}\right)$ has only the last component different from 0 , that is

$$
\theta_{2 j}\left(\mathbf{z}_{1}\right)=\left(\begin{array}{c}
0 \\
\vdots \\
0 \\
\vartheta_{2 j}\left(\mathbf{z}_{1}\right)
\end{array}\right), \forall i \in[0, \ell]
$$

Proof Assume that there exist a regular bicausal static state feedback

$$
u_{[0]}=\hat{\alpha}(\mathbf{x})+\hat{\beta}(\mathbf{x}) v_{[0]}
$$

and a bicausal change of coordinates,

$$
z_{[0]}=\left(\begin{array}{l}
z_{1,[0]} \\
z_{2,[0]}
\end{array}\right)=\varphi(\mathbf{x})
$$

with $z_{1,[0]} \in \mathbb{R}^{r}$, such that the closed-loop system in the new coordinates reads (13). Then

$$
d y_{[0]}=d \tilde{H}\left(\mathbf{z}_{1}\right) \text { and } d y_{[0]}^{(i)}=d \psi_{i}\left(\mathbf{z}_{1}\right)
$$

for $i \in[1, r-1]$. Let

$$
d z_{[0]}=\left(\begin{array}{l}
d z_{1,[0]} \\
d z_{2,[0]}
\end{array}\right)=\left(\begin{array}{l}
T_{1}(\mathbf{x}, \delta) \\
T_{2}(\mathbf{x}, \delta)
\end{array}\right) d x_{[0]}=T(\mathbf{x}, \delta) d x_{[0]}
$$

be the differential representation of the bicausal change of coordinates. Then, by assumption,

$$
\left(\begin{array}{c}
d y_{[0]} \\
\vdots \\
d y_{[0]}^{(r-1)}
\end{array}\right)=Q\left(\mathbf{z}_{1}, \delta\right) d z_{1,[0]}=\left.Q\left(\mathbf{z}_{1}, \delta\right)\right|_{z_{1,[0]}=\varphi_{1}(\mathbf{x})} T_{1}(\mathbf{x}, \delta) d x_{[0]}
$$

so that $\mathbf{Y}(\mathbf{x}, \delta)$ in (16) is given by $\mathbf{Y}(\mathbf{x}, \delta)=\bar{Q}(\mathbf{x}, \delta) T_{1}(\mathbf{x}, \delta)$. It is immediately seen that denoting by $\Delta(\mathbf{x}, \delta)$ the last $n-r$ columns of $T^{-1}(\mathbf{x}, \delta)$, then $\Delta(\mathbf{x}, \delta)$ is the right annihilator of $\mathbf{Y}(\mathbf{x}, \delta)$, has rank $n-r$ and is causal, which proves the necessity of (i). 
As for (ii), by assumption in the $z$-coordinates, and after the regular bicausal static state feedback

$$
u_{[0]}=\hat{\alpha}(\mathbf{x})+\hat{\beta}(\mathbf{x}) v_{[0]}
$$

which in the $z$-coordinates reads

$$
u_{[0]}=\tilde{\alpha}(\mathbf{z})+\tilde{\beta}(\mathbf{z}) v_{[0]},
$$

the differential representation of the $r$ th derivative of the output of the closed-loop system is given by

$$
d y_{[0]}^{(r)}=\tilde{a}\left(\mathbf{z}_{1}, \mathbf{v}, \delta\right) d z_{1,[0]}+\tilde{b}\left(\mathbf{z}_{1}, \delta\right) d v_{[0]} .
$$

Let

$$
v_{[0]}=\bar{\alpha}(\mathbf{z})+\bar{\beta}(\mathbf{z}) u_{[0]}
$$

be the inverse feedback of

$$
u_{[0]}=\tilde{\alpha}(\mathbf{z})+\tilde{\beta}(\mathbf{z}) v_{[0]} .
$$

Then

$$
\bar{\alpha}(\mathbf{z})=-\tilde{\beta}^{-1}(\mathbf{z}) \tilde{\alpha}(\mathbf{z}) \text { and } \bar{\beta}(\mathbf{z})=\tilde{\beta}^{-1}(\mathbf{z}) .
$$

Let $d v_{[0]}=\tilde{\gamma}(\mathbf{z}, \mathbf{u}, \delta) d z_{[0]}+\bar{\beta}(\mathbf{z}) d u_{[0]}$ be its differential representation and note that $d \bar{\alpha}(\mathbf{z})=\tilde{\gamma}(\mathbf{z}, 0, \delta) d z_{[0]}$. Then for the open loop system, one has that

$$
\begin{aligned}
d y_{[0]}^{(r)}= & \bar{a}(\mathbf{z}, \mathbf{u}, \delta) d z_{[0]}+\bar{b}(\mathbf{z}, \delta) d u_{[0]} \\
= & \left.\tilde{a}\left(\mathbf{z}_{1}, \mathbf{v}, \delta\right)\right|_{v_{[0]}=\bar{\alpha}(\mathbf{z})+\bar{\beta}(\mathbf{z}) u_{[0]}} d z_{1,[0]}+\tilde{b}\left(\mathbf{z}_{1}, \delta\right) \tilde{\gamma}(\mathbf{z}, \mathbf{u}, \delta) d z_{[0]} \\
& +\tilde{b}\left(\mathbf{z}_{1}, \delta\right) \bar{\beta}(\mathbf{z}) d u_{[0]} \\
= & \hat{a}(\mathbf{z}, \mathbf{u}, \delta) d z_{1,[0]}+\tilde{b}\left(\mathbf{z}_{1}, \delta\right) \tilde{\gamma}(\mathbf{z}, \mathbf{u}, \delta) d z_{[0]}+\tilde{b}\left(\mathbf{z}_{1}, \delta\right) \bar{\beta}(\mathbf{z}) d u_{[0]}
\end{aligned}
$$

which shows that

$$
\begin{aligned}
\bar{b}(\mathbf{z}, \delta) & =\tilde{b}\left(\mathbf{z}_{1}, \delta\right) \bar{\beta}(\mathbf{z}) \\
\bar{a}(\mathbf{z}, \mathbf{u}, \delta) d z_{[0]} & =\hat{a}(\mathbf{z}, \mathbf{u}, \delta) d z_{1,[0]}+\tilde{b}\left(\mathbf{z}_{1}, \delta\right) \tilde{\gamma}(\mathbf{z}, \mathbf{u}, \delta) d z_{[0]},
\end{aligned}
$$

so that

$$
\bar{a}(\mathbf{z}, 0, \delta) d z_{[0]}=\hat{a}(\mathbf{z}, 0, \delta) d z_{1,[0]}+\tilde{b}\left(\mathbf{z}_{1}, \delta\right) \tilde{\gamma}(\mathbf{z}, 0, \delta) d z_{[0]} .
$$


Recall that $b(\mathbf{x}, \delta)$ in (17) is linked to $\bar{b}(\mathbf{z}, \delta)$ computed above through the relation $b(\mathbf{x}, \delta)=\left.\bar{b}(\mathbf{z}, \delta)\right|_{z_{[0]}=\varphi(\mathbf{x})}$, so that one gets:

$$
b(\mathbf{x}, \delta)=\left.\bar{b}(\mathbf{z}, \delta)\right|_{z_{[0]}=\varphi(\mathbf{x})}=\left.\left.\tilde{b}\left(\mathbf{z}_{1}, \delta\right)\right|_{z_{1,[0]}=\varphi_{1}(\mathbf{x})} \bar{\beta}(\mathbf{z})\right|_{z_{[0]}=\varphi(\mathbf{x})}=\tilde{b}(\mathbf{x}, \delta) \beta(\mathbf{x})
$$

where $\tilde{b}_{j}(\mathbf{x})=\left.\bar{b}_{j}\left(\mathbf{z}_{1}\right)\right|_{z_{1,[0]}=\varphi_{1}(\mathbf{x})}$ so that for all $j \in[0, \ell], d \tilde{b}_{j}(\mathbf{x}) \in \Delta(\mathbf{x}, \delta)^{\perp}$, thus proving (18) and (19) in (iia).

Analogously $a(\mathbf{x}, 0, \delta)$ and $\gamma(\mathbf{x}, \delta)$ used in (20) are linked respectively to $\bar{a}(\mathbf{z}, 0, \delta)$ and $\tilde{\gamma}(\mathbf{z}, \delta)$ defined above through the relations $a(\mathbf{x}, 0, \delta)=\left.\bar{a}(\mathbf{z}, 0, \delta)\right|_{z_{[0]}=\varphi(\mathbf{x})}$ $T(\mathbf{x}, \delta)$ and

$$
\gamma(\mathbf{x}, \delta)=\left.\tilde{\gamma}(\mathbf{z}, \delta)\right|_{z_{[0]}=\varphi(\mathbf{x})} T(\mathbf{x}, \delta)
$$

It follows from (22), that

$$
\begin{aligned}
& (a(\mathbf{x}, 0, \delta)-\tilde{b}(\mathbf{x}, \delta) \gamma(\mathbf{x}, \delta)) d x_{[0]} \\
& =\left.\left((\hat{a}(\mathbf{z}, 0, \delta), 0)-\tilde{b}\left(\mathbf{z}_{1}, \delta\right) \tilde{\gamma}(\mathbf{z}, \delta)\right)\right|_{z_{[0]}=\varphi(\mathbf{x})} T(\mathbf{x}, \delta) d x_{[0]} \\
& =\left.\bar{a}(\mathbf{z}, 0, \delta)\right|_{z_{[0]}=\varphi(\mathbf{x})} d \varphi_{1}(\mathbf{x})
\end{aligned}
$$

which proves (20).

As for the sufficiency, assume that the conditions are satisfied and let the $r$ th derivative of the output be

$$
y_{[0]}^{(r)}=\bar{a}(\mathbf{x})+\sum_{j=0}^{\ell} b_{j}(\mathbf{x}) u_{[0]}(-j) .
$$

Consider the regular bicausal static state feedback

$$
u_{[0]}=\hat{\alpha}(\mathbf{x})+\hat{\beta}(\mathbf{x}) v_{[0]}=\beta^{-1}(\mathbf{x})\left(-\alpha(\mathbf{x})+v_{[0]}\right),
$$

where $\beta(\mathbf{x})$ is computed from (18), while $\alpha(\mathbf{x})$ is computed starting from $\gamma(\mathbf{x}, \delta)$ satisfying (20), with

$$
d \alpha(\mathbf{x})=\gamma(\mathbf{x}, \delta) d x_{[0]} .
$$

For the closed-loop system, the $r$ th derivative of the output becomes

$$
y_{[0]}^{(r)}=\bar{a}(\mathbf{x})+\sum_{j=0}^{\ell} b_{j}(\mathbf{x}) \beta^{-1}(\mathbf{x}(-j))\left(-\alpha(\mathbf{x}(-j))+v_{[0]}(-j)\right)
$$

Due to (18), each coefficient $b_{j}(\mathbf{x})=\tilde{b}_{j}(\mathbf{x}) \beta(\mathbf{x}(-j))$, so that 


$$
y_{[0]}^{(r)}=\bar{a}(\mathbf{x})+\sum_{j=0}^{\ell} \tilde{b}_{j}(\mathbf{x})\left(-\alpha(\mathbf{x}(-j))+v_{[0]}(-j)\right)
$$

From (i) choose a bicausal change of coordinates $z_{[0]}=\varphi(\mathbf{x})$, such that $d z_{1,0} \perp$ $\Delta(\mathbf{x}, \delta)$ with $z_{1,[0]} \in \mathrm{R}^{r}$. Then, by Lemma 1 ,

$$
d y_{[0]}^{(i)} \in \operatorname{span}_{\mathscr{K}(\delta]}\left\{d z_{1,[0]}\right\},
$$

for $i \in[0, r-1]$. Furthermore in these new coordinates the $r$ th derivative of the output of the closed-loop system is

$$
\begin{aligned}
y_{[0]}^{(r)}= & \left.\bar{a}(\mathbf{x})\right|_{x_{[0]}=\varphi^{-1}(\mathbf{z})}-\left.\sum_{j=0}^{\ell}\left(\tilde{b}_{j}(\mathbf{x}) \alpha(\mathbf{x}(-j))\right)\right|_{x_{[0]}=\varphi^{-1}(\mathbf{z})} \\
& +\left.\sum_{j=0}^{\ell} \tilde{b}_{j}(\mathbf{x})\right|_{x_{[0]}=\varphi^{-1}(\mathbf{z})} v_{[0]}(-j)
\end{aligned}
$$

Due to (19), $d \tilde{b}_{j}(\mathbf{x}) \in \Delta^{\perp}(\mathbf{x}, \delta)$, so that $\left.\tilde{b}_{j}(\mathbf{x})\right|_{x_{[0]}=\varphi^{-1}(\mathbf{z})}=\tilde{b}_{j}\left(\mathbf{z}_{1}\right)$. Finally due to (20), we get

$$
d \bar{a}(\mathbf{x})-\sum_{j=0}^{\ell}\left(\tilde{b}_{j}(\mathbf{x}) \delta^{j} d \alpha(\mathbf{x})\right) \in \Delta(\mathbf{x}, \delta)^{\perp},
$$

so that also

$$
d \bar{a}(\mathbf{x})-\sum_{j=0}^{\ell} d\left(\tilde{b}_{j}(\mathbf{x}) \alpha(\mathbf{x}(-j))\right) \in \Delta(\mathbf{x}, \delta)^{\perp},
$$

which proves that for the closed-loop system in the new coordinates

$$
y_{[0]}^{(r)}=\tilde{a}\left(\mathbf{z}_{1}\right)+\sum_{j=0}^{\ell} \tilde{b}_{j}\left(\mathbf{z}_{1}\right) v_{[0]}(-j)
$$

Due to Lemma 1, in the $z$-coordinates one has that

$$
\left(\begin{array}{c}
d y_{[0]} \\
\vdots \\
d y_{[0]}^{(r-1)}
\end{array}\right)=Q\left(\mathbf{z}_{1}, \delta\right) d z_{1,[0]}
$$


Consider the derivative of both sides of (23). Then one gets that for the closed-loop system

$$
\begin{aligned}
\left(\begin{array}{c}
d \dot{y}_{[0]} \\
\vdots \\
d y_{[0]}^{(r)}
\end{array}\right) & =\dot{Q}\left(\mathbf{z}_{1}, \delta\right) d z_{1,[0]}+Q\left(\mathbf{z}_{1}, \delta\right) d \dot{z}_{1,[0]} \\
& =P_{1}\left(\mathbf{z}_{1}, \mathbf{v}, \delta\right) d z_{1,[0]}+P_{2}\left(\mathbf{z}_{1}, \delta\right) d v_{[0]},
\end{aligned}
$$

that is

$$
Q\left(\mathbf{z}_{1}, \delta\right) d \dot{z}_{1,[0]}=\bar{P}_{1}(\mathbf{z}, \mathbf{v}, \delta) d z_{1,[0]}+P_{2}\left(\mathbf{z}_{1}, \delta\right) d v_{[0]} .
$$

The proof is carried out by contradiction, by showing that $d \dot{z}_{1,[0]}$ cannot depend on $d z_{2,[0]}$. Assume in fact that this is not the case. Then,

$$
d \dot{z}_{1,[0]}=\hat{P}_{11}(\mathbf{z}, \delta) d z_{1,[0]}+\hat{P}_{12}(\mathbf{z}, \delta) d z_{2,[0]}+\hat{P}_{13}(\mathbf{z}, \delta) d v_{[0]}
$$

Thus, premultiplying both sides by $Q\left(\mathbf{z}_{1}, \delta\right)$, one gets that

$$
\begin{aligned}
Q\left(\mathbf{z}_{1}, \delta\right) d \dot{z}_{1,[0]}= & Q\left(\mathbf{z}_{1}, \delta\right) \hat{P}_{11}(\mathbf{z}, \delta) d z_{1,[0]}+Q\left(\mathbf{z}_{1}, \delta\right) \hat{P}_{12}(\mathbf{z}, \delta) d z_{2,[0]} \\
& +Q\left(\mathbf{z}_{1}, \delta\right) \hat{P}_{13}(\mathbf{z}, \delta) d v_{[0]}
\end{aligned}
$$

which compared with (25) implies that $Q\left(\mathbf{z}_{1}, \delta\right) \hat{P}_{12}(\mathbf{z}, \delta)=0$. Since by assumption $Q\left(\mathbf{z}_{1}, \delta\right)$ is a full rank matrix, this can only happen if $\hat{P}_{12}(\mathbf{z}, \delta)=0$, which thus proves the thesis. As a consequence the system can be put in the form (13). To end the proof we have to show that the $z_{1,[0]}$ coordinates can always be chosen in order to guarantee that the $\theta_{2 j}$ 's in (13) are of the form (21). This follows immediately by considering the subsystem

$$
\begin{aligned}
\dot{z}_{1,[0]} & =\theta_{1}\left(\mathbf{z}_{\mathbf{1},[\ell]}\right)+\sum_{j=0}^{\ell} \theta_{2 j}\left(\mathbf{z}_{\mathbf{1},[\ell]}\right) v_{[0]}(-j), \\
y_{[0]} & =\tilde{H}\left(\mathbf{z}_{\mathbf{1},[\ell]}\right),
\end{aligned}
$$

whose associated differential representation (4) is characterized by

$$
g_{11}\left(\mathbf{z}_{1,[\ell]}, \delta\right)=\sum_{j=0}^{\ell} \theta_{2 j}\left(\mathbf{z}_{1,[\ell]}\right) \delta^{j}
$$

Since $d y_{[0]}^{(i)} g_{11}\left(\mathbf{z}_{1}, \delta\right)=0$ for $i \in[0, r-2]$, then

$$
\Delta\left(\mathbf{z}_{1}, \delta\right)=\operatorname{span}_{\mathscr{K}(\delta]}\left\{g_{11}\left(\mathbf{z}_{1}, \delta\right)\right\}
$$


is completely integrable and since rank $\Delta(\mathbf{x}, \delta)=1$, there exists $r-1$ exact differentials which lay in the left-annihilator. Due to Lemma 1 there exists a bicausal change of coordinates $\tilde{z}_{1,[0]}=\bar{\varphi}_{1}\left(\mathbf{z}_{1}\right)$, such that in the new coordinates

$$
\tilde{g}_{11}\left(\tilde{\mathbf{z}}_{1, \bar{\ell}]}, \delta\right)=\left(\begin{array}{c}
0 \\
\vdots \\
0 \\
*
\end{array}\right)
$$

that is

$$
\begin{aligned}
\dot{\tilde{z}}_{1,[0]} & =\tilde{\theta}_{1}\left(\tilde{\mathbf{z}}_{1,[\bar{\ell}]}\right)+\sum_{j=0}^{\bar{\ell}} \tilde{\theta}_{2 j}\left(\tilde{\mathbf{z}}_{1,[\bar{\ell}]}\right) v_{[0]}(-j), \\
y_{[0]} & =\tilde{H}\left(\tilde{\mathbf{z}}_{1,[\bar{\ell}]}\right),
\end{aligned}
$$

with

$$
\tilde{\theta}_{2 j}\left(\tilde{\mathbf{z}}_{1,[\bar{\ell}]}\right)=\left(\begin{array}{c}
0 \\
\vdots \\
0 \\
\tilde{\vartheta}_{2 j}\left(\tilde{\mathbf{z}}_{1}\right)
\end{array}\right), \quad \forall j \in[0, \bar{\ell}]
$$

which ends the proof.

We next give two examples which show how to apply the previous result.

Example 1 Consider the dynamics

$$
\begin{aligned}
\dot{x}_{1,[0]}= & x_{1,[0]} x_{1,[0]}(-1)+x_{2,[0]}-x_{1,[0]}+v_{[0]} \\
\dot{x}_{2,[0]}= & -\left(x_{1,[0]} x_{1,[0]}(-1)+x_{2,[0]}\right)^{3} \\
& -\left(x_{1,[0]} x_{1,[0]}(-1)+x_{2,[0]}-x_{1,[0]}+v_{[0]}\right) x_{1,[0]}(-1) \\
& -\left(x_{1,[0]}(-1) x_{1,[0]}(-2)+x_{2,[0]}(-1)-x_{1,[0]}(-1)+v_{[0]}(-1)\right) x_{1,[0]} \\
& +v_{[0]}(-1)
\end{aligned}
$$

with output function

$$
y_{[0]}=x_{1,[0]} x_{1,[0]}(-1)+x_{2,[0]}
$$

Then we get that the output has relative degree $r=1$ and we have that

$$
d y_{[0]}=\left(x_{1,[0]}(-1)+x_{1,[0]} \delta, 1\right) d x_{[0]} .
$$


Accordingly, the right annihilator is

$$
\Delta(\mathbf{x}, \delta)=\left(\begin{array}{c}
-1 \\
x_{1,[0]}(-1)+x_{1,[0]} \delta
\end{array}\right)
$$

which is causal. Since $d y_{[0]}$ is closed we have that $d \lambda_{1}(\mathbf{x})=d y_{[0]}$ and a possible completion to get a bicausal change of coordinates is $d z_{2,[0]}=d x_{1,[0]}$. One thus has that in the coordinates

$$
d z_{[0]}=\left(\begin{array}{rr}
x_{1,[0]}(-1)+x_{1,[0]} \delta & 1 \\
1 & 0
\end{array}\right) d x_{[0]} \rightarrow z_{[0]}=\left(\begin{array}{c}
x_{1,[0]} x_{1,[0]}(-1)+x_{2,[0]} \\
x_{1,[0]}
\end{array}\right)
$$

the system reads

$$
\dot{z}_{1,[0]}=-z_{1,[0]}^{3}+v_{[0]}(-1), \quad \dot{z}_{2,[0]}=z_{1,[0]}-z_{2,[0]}+v_{[0]}, \quad y_{[0]}=z_{1,[0]} .
$$

Example 2 Consider again the dynamics (1), but with output function

$$
y_{[0]}=x_{1,[0]} x_{1,[0]}(-1)+x_{2,[0]} x_{2,[0]}(-1)
$$

In this case,

$$
d y_{[0]}=\left(x_{1,[0]}(-1)+x_{1,[0]} \delta x_{2,[0]}(-1)+x_{2,[0]} \delta\right),
$$

which is still closed. However the right annihilator in this case is

$$
\Delta(\mathbf{x}, \delta)=\left(\begin{array}{c}
-\frac{x_{2,[0]}(-1)}{x_{1,[0]}(-1)} b_{0}-x_{2,[0]}(+1) \delta \\
b_{0}+x_{1,[0]}(+1) \delta
\end{array}\right)
$$

with

$$
b_{0}=\frac{x_{1,[0]} x_{2,[0]}(+2)-x_{2,[0]} x_{1,[0]}(+2)}{x_{2,[0]}(+1) x_{1,[0]}(-1)-x_{1,[0]}(+1) x_{2,[0]}(-1)} x_{1,[0]}(-1) .
$$

Obviously the generator of $\Delta(\mathbf{x}, \delta)$ can never be chosen causal, so that there is no causal function $\lambda(\mathbf{x})$ such that $d \mathrm{x}_{[0]} \in \operatorname{span}_{\mathscr{K}(\delta]}\left\{d y_{[0]}, d \lambda\right\}$.

We end this section by giving the conditions under which the unobservable dynamics in (13) can be chosen in order to be independent of the control. The following result holds true.

Theorem 5 Given system (1), with output $y_{[0]}$ characterized by the well defined relative degree $r$, there exist a regular bicausal static state feedback $u_{[0]}=\alpha(\mathbf{x})+$ $\beta(\mathbf{x}) v_{[0]}$, and a bicausal change of coordinates 


$$
z_{[0]}=\left(\begin{array}{l}
z_{1,[0]} \\
z_{2,[0]}
\end{array}\right)=\varphi(\mathbf{x})
$$

with $z_{1,[0]} \in \mathbb{R}^{r}$, such that in the new coordinates the closed-loop system reads as (13) with $\eta_{2 j}=0, j \in[0, \ell]$, if and only if the conditions of Theorem 4 are satisfied, and additionally,

(a) given the differential representation (4), associated with the system,

$$
\Delta(\mathbf{x}, \delta)=\operatorname{span}_{\mathscr{K}(\delta]}\left\{g_{1}(\mathbf{x}, \delta)\right\}
$$

is completely integrable, that is, there exists an index $\gamma$ such that $\rho_{\gamma}-\rho_{\gamma-1}=1$; (b) in the z-coordinates in which the system reads (13) with $\theta_{2 j}$ of the form (21), denoting by $\eta_{2}(\mathbf{z}, \delta)=\sum_{j=0}^{\ell} \eta_{2 j}(\mathbf{z}) \delta^{j}$ and by $\vartheta_{2}\left(\mathbf{z}_{1}, \delta\right)=\sum_{j=0}^{\ell} \vartheta_{2 j}\left(\mathbf{z}_{1}\right) \delta^{j}$, then

$$
\eta_{2}(\mathbf{z}, \delta)=\left(\begin{array}{c}
m_{1}(\mathbf{z}, \delta) \\
\vdots \\
m_{n-r}(\mathbf{z}, \delta)
\end{array}\right) \vartheta_{2}\left(\mathbf{z}_{1}, \delta\right)
$$

Proof The proof of the necessity is immediate if one considers that, condition (a) ensures that there exist $n-1$ exact differentials in the left kernel of $g_{1}(\mathbf{x}, \delta)$, which are the candidates for the bicausal change of coordinates. The second condition is necessary to guarantee that one can find a bicausal change of coordinates which achieves the result by transforming only the last $n-r$ components, since the first $r$ ones are related to the input output behavior.

As for the sufficiency, suppose for simplicity that the system has already been transformed in the form (13), with $\theta_{2 j}$ of the form (21). Since (a) holds true one can find $n-1$ exact differentials which lie in the left kernel of

$$
\Delta(\mathbf{x}, \delta)=\operatorname{span}_{\mathscr{K}(\delta]}\left\{g_{1}(\mathbf{z}, \delta)\right\} .
$$

Starting with the computation of such exact differentials at the first step one gets

$$
d z_{11,[0]}, \cdots, d z_{1 r-1,[0]}
$$

We now have to show the form of the last $n-r$ exact differentials. To this end note that, due to b), a basis for $\Delta(\mathbf{x}, \delta)^{\perp}$ is

$$
\Delta(\mathbf{x}, \delta)^{\perp}=\operatorname{span}_{\mathscr{K}(\delta]}\left\{d z_{1 i,[0]}, i \in[1, r-1], d z_{2 j,[0]}-m_{j}(\mathbf{z}, \delta) d z_{1} r,[0], j \in[1, n-r]\right\}
$$

Since the previous collection of one-forms is closed, then any other collection which generates $\Delta(\mathbf{x}, \delta)^{\perp}$ must be obtained through a unimodular matrix. Let $d \varphi_{2 j}(\mathbf{z})$, $j \in[1, \cdots, n-r]$, be the set of exact differentials such that

$$
\Delta(\mathbf{x}, \delta)^{\perp}=\operatorname{span}_{\mathscr{K}(\delta]}\left\{d z_{1 i,[0]}, i \in[1, r-1], d \varphi_{2 j}(\mathbf{z}), j \in[1, n-r]\right\}
$$


then

$$
\left(\begin{array}{c}
d z_{11,[0]} \\
\vdots \\
d z_{1 r-1,[0]} \\
d \varphi_{21}(\mathbf{z}) \\
\vdots \\
d \varphi_{2 n-r}(\mathbf{z})
\end{array}\right)=\left(\begin{array}{cc}
I & 0 \\
T_{21}(\mathbf{z}, \delta) & T_{22}(\mathbf{z}, \delta)
\end{array}\right)\left(\begin{array}{c}
d z_{11,[0]} \\
\vdots \\
d z_{1 r-1,[0]} \\
d z_{21,[0]}-m_{1}(\mathbf{z}, \delta) d z_{1} r,[0] \\
\vdots \\
d z_{2 n-r,[0]}-m_{n-r}(\mathbf{z}, \delta) d z_{1} r,[0]
\end{array}\right)
$$

where $T_{22}(\mathbf{z}, \delta)$ is unimodular. Consequently the change of coordinates

$$
\left(\begin{array}{c}
d z_{11,[0]} \\
\vdots \\
d z_{1} r,[0] \\
d \varphi_{21}(\mathbf{z}) \\
\vdots \\
d \varphi_{2 n-r}(\mathbf{z})
\end{array}\right)
$$

is bicausal.

\subsection{Normal Form}

In this section we analyze the conditions under which there exist a bicausal change of coordinates and a regular bicausal static state feedback thanks to which the closedloop system can be decomposed in the form (13), with a linear input output behavior, that is the closed-loop system displays a linear input-output relation and reads in the new coordinates

$$
\begin{aligned}
\dot{z}_{1,[0]} & =\sum_{j=0}^{\ell} A_{j} z_{1,[0]}(-j)+\sum_{j=0}^{\ell} B_{j} v_{[0]}(-j), \\
\dot{z}_{2,[0]} & =\eta_{1}\left(\mathbf{z}_{[\ell]}\right)+\sum_{j=0}^{\ell} \eta_{2 j}\left(\mathbf{z}_{[\ell]}\right) v_{[0]}(-j) \\
y_{[0]} & =\sum_{j=0}^{\ell} C_{j} z_{1,[0]}(-j),
\end{aligned}
$$

with $z_{1,[0]} \in \mathbb{R}^{r}, r$ being the relative degree of the output.

Of course, in this case the conditions of the previous Theorem 4 must be satisfied together with some new additional ones. The following result holds true. 
Theorem 6 Given system (1), with output $y_{[0]}$ characterized by the well defined relative degree $r$, there exist a regular bicausal static state feedback

$$
u_{[0]}=\hat{\alpha}(\mathbf{x})+\hat{\beta}(\mathbf{x}) v_{[0]},
$$

and a bicausal change of coordinates

$$
z_{[0]}=\left(\begin{array}{l}
\mathbf{z}_{1,[0]} \\
\mathbf{z}_{2,[0]}
\end{array}\right)=\varphi(\mathbf{x})
$$

with $z_{1,[0]} \in \mathbb{R}^{r}$, such that in the new coordinates the closed-loop system is in the Normal Form (26) if and only if the conditions of Theorem 4 are satisfied and additionally

(i') $\mathbf{Y}(\mathbf{x}, \delta)$ in (16) can be factorized as $\mathbf{Y}(\mathbf{x}, \delta)=Q(\delta) T_{1}(\mathbf{x}, \delta)$, with $Q(\delta)$ a matrix with coefficients in $R(\delta]$, and the submodule generated by $T_{1}(\mathbf{x}, \delta) d \mathrm{x}_{[0]}$ is left-closed.

(ii') $\tilde{b}(\mathbf{x}, \delta)$ in (18) is independent of $\mathbf{x}$ that is $\tilde{b}(\mathbf{x}, \delta)=\tilde{b}(\delta)$. Accordingly, (19) is always satisfied.

(iii') the exact differential $\gamma(\mathbf{x}, 0, \delta) d x_{[0]}=d \alpha(\mathbf{x})$ must satisfy

$$
(a(\mathbf{x}, 0, \delta)-\tilde{b}(\delta) \gamma(\mathbf{x}, \delta)) d x_{[0]} \in \operatorname{span}_{R(\delta]}\left\{d \lambda_{1}, \cdots, d \lambda_{r}\right\}
$$

The coordinates $z_{1}=\left(\lambda_{1}, \cdots, \lambda_{r}\right)^{T}$ can be chosen in such a way that the $B_{j}$ 's in the closed-loop system (26), only have the last component different from 0 , that is,

$$
B_{j}=\left(\begin{array}{c}
0 \\
\vdots \\
0 \\
b_{j r}
\end{array}\right), \quad j \in[0, \ell]
$$

Proof Of course the conditions of Theorem 4 must be satisfied, since the structure (26) represents a special case of the structure (13). The necessity and sufficiency of the additional conditions are proved hereafter. As for the necessity, if the system can be put in the form (26), then one gets that in the good coordinates

$$
\left(\begin{array}{c}
d y_{[0]} \\
\vdots \\
d y_{[0]}^{(r-1)}
\end{array}\right)=\left(\begin{array}{c}
C(\delta) \\
\vdots \\
C(\delta) A^{r-1}(\delta)
\end{array}\right) d z_{1,[0]}=Q(\delta) T_{1}(\mathbf{x}, \delta) d x_{[0]}
$$


which proves the necessity of (i'). Furthermore, for the closed-loop system one must have that

$$
d y_{[0]}^{(r)}=\tilde{a}(\delta) d z_{1,[0]}+\tilde{b}(\delta) d v_{[0]}
$$

For any regular bicausal static state feedback $v_{[0]}=\bar{\alpha}(\mathbf{z})+\bar{\beta}(\mathbf{z}) u_{[0]}$, one has that

$$
d v_{[0]}=\bar{\gamma}(\mathbf{z}, \mathbf{u}, \delta) d z_{[0]}+\bar{\beta}(\mathbf{z}) d u_{[0]},
$$

so that

$$
\begin{aligned}
d y_{[0]}^{(r)} & =\tilde{a}(\delta) d z_{1,[0]}+\tilde{b}(\delta) \bar{\gamma}(\mathbf{z}, \mathbf{u}, \delta) d z_{[0]}+\tilde{b}(\delta) \bar{\beta}(\mathbf{z}) d u_{[0]} \\
& =\tilde{a}(\mathbf{z}, \mathbf{u}, \delta) d z_{[0]}+\tilde{b}(\delta) \bar{\beta}(\mathbf{z}) d u_{[0]}
\end{aligned}
$$

which immediately proves (ii') and (iii').

As for the sufficiency, since the conditions of Theorem 4 are satisfied, in the new coordinates the system reads as (13).

Due to (i'), we can take as $z_{1,[0]}$ coordinates $z_{1,[0]}=\left(\lambda_{1}(\mathbf{x}), \cdots, \lambda_{r}(\mathbf{x})\right)^{T}$.

Taking $u_{[0]}=\beta^{-1}(\mathbf{x})\left(-\alpha(\mathbf{x})+v_{[0]}\right)$ as the feedback law, where $\beta(\mathbf{x})$ and $\alpha(\mathbf{x})$ are chosen as in Theorem 4 one gets that for the closed loop system, the $r$ th derivative of the output becomes

$$
y_{[0]}^{(r)}=\bar{a}(\mathbf{x})+\sum_{j=0}^{\ell} b_{j}(\mathbf{x}) \beta^{-1}(\mathbf{x}(-j))\left(-\alpha(\mathbf{x}(-j))+v_{[0]}(-j)\right)
$$

Due to (ii') and (iii'), each coefficient $b_{j}(\mathbf{x})=\tilde{b}_{j} \beta(\mathbf{x}(-j))$, so that

$$
y_{[0]}^{(r)}=\bar{a}(\mathbf{x})+\sum_{j=0}^{\ell} \tilde{b}_{j}\left(-\alpha(\mathbf{x}(-j))+v_{[0]}(-j)\right)
$$

Consequently, its differential representation is

$$
\begin{aligned}
d y_{[0]}^{(r)} & =d \bar{a}(\mathbf{x})-\sum_{j=0}^{\ell} \tilde{b}_{j} d \alpha(\mathbf{x}(-j))+\sum_{j=0}^{\ell} \tilde{b}_{j} d v_{[0]}(-j) \\
& =\tilde{a}(\delta) d z_{1,[0]}+\tilde{b}(\delta) d v_{[0]}
\end{aligned}
$$

Furthermore, from (i'),

$$
\left(\begin{array}{c}
d y_{[0]} \\
\vdots \\
d y_{[0]}^{(r-1)}
\end{array}\right)=Q(\delta) T_{1}(\mathbf{x}, \delta) d x_{[0]}=Q(\delta) d z_{1,[0]}
$$


As a consequence,

$$
\left(\begin{array}{c}
d \dot{y}_{[0]} \\
\vdots \\
d y_{[0]}^{(r)}
\end{array}\right)=Q(\delta) d \dot{z}_{1,[0]}=P_{1}(\delta) d z_{1,[0]}+P_{2}(\delta) d u_{[0]}
$$

Multiply both sides on the left by the adjugate matrix $[Q(\delta)]^{(a)}$ of $Q(\delta)$. Since

$$
[Q(\delta)]^{(a)} Q(\delta)=q_{o}(\delta) I,
$$

where $q_{0}$ is the determinant of $Q(\delta)$ and $I$ is the identity matrix, we get

$$
q_{o}(\delta) d \dot{z}_{1,[0]}=[Q(\delta)]^{(a)} P_{1}(\delta) d z_{1,[0]}+[Q(\delta)]^{(a)} P_{2}(\delta) d u_{[0]}
$$

which immediately implies (from the identity of polynomials) that

$$
d \dot{z}_{1,[0]}=A(\delta) d z_{1,[0]}+B(\delta) d u_{[0]}
$$

as well as that $d y_{[0]}=C(\delta) d z_{1,[0]}$. This ends the proof.

In this case, one may also investigate under which conditions the unobservable dynamics is independent of the control variable. The generalization of Theorem 5 is straightforward. The following result, whose proof is omitted since it follows the same lines as the proof of Theorem 5, holds:

Theorem 7 Given system (1), with output $y_{[0]}$ characterized by the well defined relative degree $r$, there exist a regular bicausal static state feedback

$$
u_{[0]}=\hat{\alpha}(\mathbf{x})+\hat{\beta}(\mathbf{x}) v_{[0]},
$$

and a bicausal change of coordinates

$$
z_{[0]}=\left(\begin{array}{l}
z_{1,[0]} \\
z_{2,[0]}
\end{array}\right)=\varphi(\mathbf{x})
$$

with $z_{1,[0]} \in \mathbb{R}^{r}$, such that in the new coordinates the closed-loop system reads as (26) with $\eta_{2 j}=0, j \in[0, \ell]$, if and only if the conditions of Theorem 6 are satisfied and additionally

(a) given the differential representation (4), associated to the system, $\Delta(\mathbf{x}, \delta)=$ $\operatorname{span}_{\mathscr{K}(\delta]}\left\{g_{1}(\mathbf{x}, \delta)\right\}$ is completely integrable, that is there exists an index $\gamma$ such that $\rho_{\gamma}-\rho_{\gamma-1}=1$; and

(b) in the $z$ coordinates in which the system is (26) with $B_{j}$ of the form (28), we have 


$$
\eta_{2}(\mathbf{z}, \delta)=\left(\begin{array}{c}
m_{1}(\mathbf{z}, \delta) \\
\vdots \\
m_{n-r}(\mathbf{z}, \delta)
\end{array}\right) b(\delta)
$$

where $\eta_{2}(\mathbf{z}, \delta)=\sum_{j=0}^{\ell} \eta_{2 j} \delta^{j}$ and $b(\delta)=\sum_{j=0}^{\ell} b_{j} \delta^{j}$.

\section{Some Concluding Remarks}

Various normal forms have been derived for single-output time-delay systems. Some of those normal forms include candidates for defining the so-called zero dynamics. However, as already underlined in [8], the extension of the concept of zero dynamics to nonlinear systems with delays is not straightforward. As a matter of fact, in the delay-free case, zeroing the output, which has by assumption relative degree $r$, leads to the computation of a residual dynamics, called zero dynamics, which has dimension $n-r$, and in the case of single-input single-output systems, is independent of the control $u$. As shown in [10], this property has some important consequences on the stabilization procedure, since if the zero dynamics is locally asymptotically stable, then one may choose the input in order to satisfy some requirements on the input-output behavior. In the case of delay systems, instead, the zero dynamics may have a dimension greater than $n-r$ as shown in [8]. However if the conditions of Theorem 4 (respectively Theorem 6) hold, then the system can be split, as already noted, into two subsystems, the first one representing the input-output behavior, and the second one influenced by the control and the state variable of the first subsystem. The stability properties of this last subsystem become important when designing feedback laws, to guarantee a certain required input-output behavior.

\section{References}

1. Califano, C., Marquez-Martinez, L., Moog, C.H.: Extended lie brackets for nonlinear timedelay systems. IEEE Trans. Autom. Control 56(9), 2213-2218 (2011)

2. Califano, C., Marquez-Martinez, L., Moog, C.H.: Linearization of time-delay systems by inputoutput injection and output transformation. Automatica 49(6), 1932-1940 (2013)

3. Califano, C., Monaco, S., Normand-Cyrot, D.: On the discrete-time normal form. IEEE Trans. Autom. Control 43(11), 1654-1658 (1998)

4. Califano, C., Moog, C.H.: De l'existence de la forme normale pour les systemes non lineaires a retards. In: Proceedings of Conference Internationale Francophone d'Automatique, pp. 37-42 (2012)

5. Califano, C., Moog, C.H.: Coordinates transformations in nonlinear time-delay systems. In: Proceedings of the 53rd IEEE Conference on Decision and Control, pp. 475-480 (2014)

6. Garate-Garcia, A., Marquez-Martinez, L., Cuesta-Garcia, J., Garcia-Ramirez, E.: A computer algebra system for analysis and control of nonlinear time-delay systems. Adv. Eng. Softw. 65, 138-148 (2013) 
7. Germani, A., Manes, C., Pepe, P.: Linearization of input-output mapping for nonlinear delay systems via static state feedback. In: Proceedings of the IEEE-IMACS Conference on Computer Engineering in System Applications, pp. 599-602 (1996)

8. Germani, A., Manes, C., Pepe, P.: Input-output linearization with delay cancellation for nonlinear delay systems: the problem of the internal stability. Int. J. Robust Nonlinear Control 13(9), 909-937 (2003)

9. Gu, K., Kharitonov, V., Chen, J.: Stability of Time-Delay Systems. Birkhauser, Boston (2003)

10. Isidori, A.: Nonlinear Control Systems, 3rd edn. Springer, New York (1995)

11. Marquez-Martinez, L., Moog, C.H., Velasco-Villa, M.: Observability and observers for nonlinear systems with time delay. Kybernetika 38(4), 445-456 (2002)

12. Michiels, W., Niculescu, S-I.: Stability and Stabilization of Time-Delay Systems. An Eigenvalue-Based Approach. SIAM, Philadelphia (2007). (Advances in Design and Control, 12)

13. Oguchi, T.: A finite spectrum assignment for retarded non-linear systems and its solvability condition. Int. J. Control 80(6), 898-907 (2007)

14. Pepe, P., Jiang, Z.-P.: A Lyapunov Krasovskii methodology for ISS and iISS of time-delay systems. Syst. Control Lett. 55(12), 1006-1014 (2006)

15. Xia, X., Marquez-Martinez, L., Zagalak, P., Moog, C.H.: Analysis of nonlinear time-delay systems using modules over non-commutative rings. Automatica 38(9), 1549-1555 (2002)

16. Zheng, G., Barbot, J., Boutat, D.: Identification of the delay parameter for nonlinear time-delay systems with unknown inputs. Automatica 49(6), 1755-1760 (2013) 